(C) Facultad de Ciencias Biológicas UNMSM

\title{
Evaluación de Spalangia endius y Muscidifurax sp. (Hymenoptera, Pteromalidae) como controladores de Musca domestica en el Perú \\ Evaluation of Spalangia endius and Muscidifurax sp. (Hymenoptera, Pteromalidae) as controller of Musca domestica en el Peru
}

\author{
Edgar Inciso ${ }^{1}$ y Julia Castro ${ }^{2}$
}

\begin{abstract}
Programa Nacional de Control Biológico. Servicio Nacional de Sanidad Agraria, SENASA, Laboratorio de Insectos.

${ }^{2}$ Facultad de Ciencias Biológicas, Laboratorio de Control de Artrópodos y Vectores. Universidad $\mathrm{Na}$ cional Mayor de San Marcos.

Email Julia Castro: jcastroh@unmsm.edu.pe
\end{abstract}

\section{Resumen}

En el presente trabajo se comparó la actividad parasitoide de las microavispas Spalangia endius y Muscidifurax sp. sobre el estadio pupal de Musca domestica. La mejor temperatura para la crianza fue de $25^{\circ} \mathrm{C}$. El ciclo de $\mathrm{S}$. endius se completó en 22,6 días, mientras en Muscidifurax sp. en 14,8 días. La duración del ciclo fue inversamente relacionada con la temperatura, siendo mayor en las hembras que en los machos. La longevidad fue similar en ambas especies de microavispas (20,7 días en S. endius y 18,6 días en Muscifurax sp.).La oviposicion de $S$. endius a los 15 días fue sobre 175 pupas de $M$. domestica, valor mayor que Muscidifurax que parasitó 140 pupas en 16 días. En ambas especies la mayor postura ocurrió al tercer día. Las pupas de $M$. domestica de dos días de maduración fueron las mas parasitadas con $66,4 \%$ de parasitismo efectivo por $S$. endius y $60,2 \%$ por Muscidifurax sp. El número óptimo de pupas de $M$. domestica fue 10 (10:1). Se concluye que en condiciones de laboratorio, Spalangia endius puede ser un eficiente controlador biológico de pupas de Musca domestica.

Palabras clave: parasitoides, Spalangia endius, Muscidifurax sp., Musca domestica

\section{Abstract}

In this work we compared the parasitoid activity of the microhymenopterans Spalangia endius and Muscidifurax sp. on the pupae of Musca domestica. The better temperature for the reared was of $25^{\circ} \mathrm{C}$. The cycle of $\mathrm{S}$. endius was completed in 22,6 days, while in Muscidifurax sp. in 14,8 days. The cycle duration was inversely related to temperature, in the females was highest than males. The longevity was similar in both microhymenopterans species $(20,7$ days in $S$. endius and 18,6 days in Muscifurax sp.). Spalangia endius oviposited on 175 pupae of $M$. domestica in 15 days; this value was highest than Muscidifurax that oviposited 140 pupae in 16 days. In both species the highest posture occurred in the third day. Musca domestica pupae with two maturation days were the more parasited; S. endius with $66,4 \%$ and Muscidifurax sp. with $60,2 \%$ of effective parasitism. The better number of $M$. domestica pupae was 10 (10:1). We conclude that under laboratory conditions, $S$. endius can be an efficient biological controller of pustules of $M$. domestica.

Keywords: parasitoid, Spalangia endius, Muscidifurax sp., Musca domestica

\section{Introducción}

Musca domestica, es un insecto que por su biología y hábitos domésticos, la convierten en un vector de importancia en salud pública y veterinaria. Los métodos químicos tradicionales para su control no han sido efectivos; frente a ello la aplicación de microavispas Spalangia endius y Muscidifurax sp. con capacidad parasitoide, surge como una alternativa para reducir las poblaciones de moscas.

Musca domestica vive en contacto cercano con el hombre y debido a esta estrecha relación la convierte en una seria amenaza para transmitir enfermedades comprometiendo el bienestar de la humanidad, debido a que transportan organismos patógenos sobre su superficie, así como en el interior de la cavidad intestinal y los diseminan en los vómitos, regurgitaciones y excrementos convirtiéndolos en eficientes vectores mecánicos de enfermedades.

La ganadería intensiva, las industrias alimentarías, los cultivos industriales y hortícola son afectadas por esta plaga y además condiciona un estado de stress tanto en las personas como en los animales, trayendo como consecuencia merma en la productividad y rendimiento de los individuos afectados (Vergara y Jiménez, 1995)

El hombre ha utilizado principalmente el control químico para poderlas combatir, lo que ha ocasionado problemas de resis- tencia, evasión de los lugares de reposo, así como un incremento de los costos de producción, es por eso que se vienen buscando métodos alternativos de control, debido a que las moscas han manifestado mayor capacidad de resistencia a los insecticidas por las continuas aplicaciones, agravando el problema económico y la contaminación del medio ambiente.

Frente al uso desmedido de plaguicidas, surge como alternativa de control biológico, la utilización de microavispas con capacidad parasitoide del estado pupal de Musca domestica (Ortiz y Torres, 1983). El uso de microavispas de la familia Pteromalidae, surge como una alternativa económica para el control de moscas debido a su actividad de ovipositar en el interior de las pupas de $M$. domestica, destruyéndolas antes que estas se conviertan en adultos, reduciendo de esta manera las poblaciones de moscas (Zamora, 1996).

Debido a la efectividad reportada de las microavispas y por los escasos estudios realizados en el país se planteó evaluar en el laboratorio la actividad parasitaria de las microavispas sobre pupas de Musca domestica y determinar los factores que aseguren el optimo ciclo vital de los parasitoides, así como evaluar su capacidad de oviposición. 


\section{Material y Métodos}

Crianza y mantenimiento de los parasitoides. Los parasitoides fueron recuperados a partir de pupas de $M$. doméstica colectados de los establos de la Universidad Agraria La Molina., con ayuda de cernidores y luego fueron transportadas al laboratorio de Control de artropodos y vectores de la Facultad de CC BB, para la emergencia de los adultos. La población de moscas emergidas fue utilizada para la crianza masiva de los hospederos. Los parasitoides fueron identificados como Spalangia endius y Muscidifurax sp. y fueron proporcionados por el Programa Nacional de Control Biológico del SENASA. La crianza se realizó en jaulas de dos mangas y los ensayos se hicieron en oscuridad. En dichas jaulas, se colocaron diariamente, pupas frescas de mosca para ser parasitadas, las que previamente fueron limpiadas para la emergencia de los nuevos parasitoides. A las dos semanas, las pupas fueron limpiadas con la ayuda de un ventilador para eliminar las moscas muertas emergidas y las exuvias pupales de donde emergieron las moscas de las pupas no parasitadas, las mismas que no debieron rebasar el 20\% del total de pupas expuestas al parasitismo. Los parasitoides adultos, machos y hembras, a los pocos minutos de emerger ya estaban capacitados para copular y se les alimentó con miel de abeja sin diluir. Para continuar con el ciclo, se les expuso nuevamente pupas frescas y luego se obtuvo una nueva generación de parasitoides.

Crianza y mantenimiento de Musca domestica. La crianza se realizó en dos jaulas grandes de dos mangas para las moscas adultas. En el interior se colocó diariamente el alimento consistente en miel de abeja en una dilución al 10\%, embebida en algodón, además de leche en polvo para mejorar su oviposición y dieta especial con salvado de trigo fermentado.

Después de la copula, la mosca adulta realizó la postura de huevos, los que fueron colocados en masas blanquecinas sobre el sustrato preparado con salvado de trigo fermentado y separados en varias placas. Las placas fueron renovadas cada 24 horas el quinto día. Después los huevos fueron sembrados en la dieta de las larvas, para su eclosión, teniendo en cuenta la temperatura, la humedad relativa y la luz.

Las larvas pasaron por 3 estadios en un lapso de 5 a 7 días, después se trasladó la dieta a un bastidor con malla de plástico de $2 \mathrm{~mm}$, el que fue colocado sobre una bandeja de plástico que contenía una delgada capa de arena limpia, para que las larvas ingresen a la arena y empupen en un lapso de 24 a 48 horas. Luego, se tamizó la arena obteniéndose pupas limpias.

Infección experimental sobre los estadíos pupales. La aplicación de los parasitoides a los puparios de $M$. doméstica fue similar para S. endius y Muscidifurax sp. Las pupas de 24--48 horas, fueron colocadas en vasos de plástico cubiertos con organza junto con los parasitoides machos y hembras (en proporción 1:1), junto con el alimento (miel de abeja sin diluir) y el ambiente de trabajo se mantuvo en oscuridad. Las pupas de donde no emergieron moscas fueron separadas ya que también habían sido parasitadas. Después de cumplir con su ciclo biológico, las avispas emergieron de los puparios y se procedió a contabilizar los parasitoides emergidos por especie.

Influencia de la temperatura en la crianza de los parasitoides (ciclo biológico y longevidad). Se desarrollo el ciclo de vida completo de Spalangia endius y Muscidifurax sp. a diferentes temperaturas: $15,20,25$ y $28^{\circ} \mathrm{C}$, utilizando una población de 100 pupas de Musca domestica para cada temperatura. Se realizaron 5 replicas por cada temperatura.

Capacidad de oviposición diaria de S. endius y Muscidifurax sp. en condiciones de laboratorio. Vasos de 70 $\mathrm{ml}$ de capacidad fueron utilizados para este experimento, en cada uno se colocaron 20 pupas de $M$. domestica con una pareja de parasitoides recién emergidos, los recipientes fueron cubiertos con organza y sujetados con liga, y se los alimentó con finas pinceladas de miel de abeja. Se realizaron 10 repeticiones para obtener pupas parasitadas. Se trabajó en condiciones de oscuridad (fotoperíodo: $0-24$ horas)y a $25^{\circ} \mathrm{C}$.

Los cambios de hospederos se efectuaron cada 24 horas, durante la longevidad de las hembras. Los machos muertos antes que las hembras fueron reemplazados para mantener las parejas, y se anotaron el número de parasitoides emergidos.

Determinación del periodo pupal óptimo de Musca doméstica para el parasitismo. Se realizaron observaciones para determinar el día en que la pupa era parasitada por el parasitoide. En cada vaso se tomaron grupos de 50 pupas con edades de 1, 2, 3 y 4 días las que fueron expuestas por 24 horas y a $25^{\circ} \mathrm{C}$ al parasitismo de las avispas hembras a quienes se les alimentó diariamente con miel de abeja. Se hizo un seguimiento a las pupas de las moscas, de donde emergieron posteriormente los parasitoides.

Determinación del número óptimo de pupas. Se trabajó con las siguientes relaciones de parasitación: 1:1, 1:5, 1:10, 1:15, 1:20,1:25, 1:30, 1:35, 1:40 y 1:50, colocando para cada relación una pareja de parasitoides y el correspondiente numero de pupas. Los machos muertos, antes que las hembras, fueron reemplazados para mantener las parejas. Cada grupo fue observado hasta la emergencia del parasitoide y se registró el número de pupas parasitadas por hembra.

Análisis estadístico. Para el cálculo de los estadísticos descriptivos e inferenciales se utilizó el paquete SPSS 7.5 en español para Window 98.

Un análisis de regresión y análisis de varianza (ANDEVA) fue aplicado para todos los ensayos. Establecida la significancia estadística del ANDEVA; se realizó la prueba de Tukey $(\mathrm{p}<0,05)$ y el diseño estadístico empleado fue un DBCR (Diseño en bloques completamente randomizado). También se utilizó el método de

Tabla 1. Prueba de Tukey considerando el tipo de microavispa y a diferentes temperaturas

\begin{tabular}{lccccccc}
\hline $\begin{array}{l}\text { DHS de Tukey a,b } \\
\text { especie-temperatura }\end{array}$ & $\mathbf{N}$ & $\mathbf{1}$ & $\mathbf{2}$ & $\mathbf{3}$ & $\mathbf{4}$ & $\mathbf{5}$ & $\mathbf{6}$ \\
\hline Muscidifurax-28 & 10 & 2,60 & & & & \\
Muscidifurax-25 $^{\circ}$ & 10 & 2,76 & & & \\
Spalangia-28 $^{\circ}$ & 10 & & 2,94 & & & \\
Muscidifurax-20 $^{\circ}$ & 10 & & 3,02 & & & \\
Spalangia-25 $^{\circ}$ & 10 & & & 3,15 & & \\
Muscidifurax-15 $^{\circ}$ & 10 & & & 3,27 & & \\
Spalangia-20 & 10 & & & & 3,48 & \\
Spalangia-15 & 10 & & & & & 3,85 \\
Significación & & 1,0 & 1,0 & 1,0 & 1,0 & 1,0 & 1,0 \\
\hline
\end{tabular}

Se muestran las medidas para los grupos en subconjuntos homogeneos. Basado en la suma de cuadrados tipo III El termino error es la Media cuadrática (Error) $=0,007$ a. Usa el tamaño muestreal de la media armónica $=10000$ b. Alfa $=0,05$ 
Tabla 2. Duración promedio del ciclo biológico en días $(X)$ a diferentes temperaturas consideradas en los experimentos con Spalangia endius y Muscidifurax sp. $\mathrm{X}=$ días promedio, DS= Desviación Standard

\begin{tabular}{lcccc}
\hline Temperatura & \multicolumn{2}{c}{ Spalangia endius } & \multicolumn{2}{c}{ Muscidifurax sp. } \\
$\left({ }^{\circ} \mathrm{C}\right)$ & X & DS & X & DS \\
\hline 15 & 46,20 & $\pm 4,13$ & 25,30 & $\pm 1,83$ \\
20 & 31,60 & $\pm 2,50$ & 19,60 & $\pm 1,58$ \\
25 & 22,60 & $\pm 2,84$ & 14,80 & $\pm 1,40$ \\
28 & 17,90 & $\pm 1,66$ & 12,50 & $\pm 0,85$ \\
\hline
\end{tabular}

gráficos a través de medias marginales. La evaluación de S. endius fue 15 días y Muscidifurax sp. fue 16 dias, ambas por 10 repeticiones. Los datos fueron transformados previamente usando: logaritmo $(x+1)$. De los resultados del ANDEVA se hizo la prueba de Tukey $(\mathrm{p}<0,05)$.

\section{Resultados y discusión}

Influencia de la temperatura en el ciclo biológico y en la longevidad de los parasitoides

El ciclo biológico de los parasitoides. La duración del ciclo biológico de ambas microavispas tiende a disminuir cuando se incrementan las temperaturas, lo que se evidenció con las ecuaciones de regresión lineal siguientes: $S$. endius $(\mathrm{Y}=53,05-0,97 \mathrm{X} ; \mathrm{F}=$ 35,86; $\mathrm{gl}=1$ y 2; $\left.\mathrm{R}^{2}=0,95\right)$; Muscidifurax sp. $(\mathrm{Y}=28,85-0,98 \mathrm{X}$; $\mathrm{F}=61,84 ; \mathrm{gl}=1$ y $\left.2 ; \mathrm{R}^{2}=0,97\right)$, donde $\mathrm{Y}=$ duración en días del ciclo biológico y $\mathrm{X}=$ temperatura de ensayo. El ANDEVA resultó estadísticamente significativo al evaluar la duración del ciclo biológico de los dos parasitoides a las cuatro temperaturas experimentales $(\mathrm{F}=217,31 ; \mathrm{gl}=7$ y $72 ; \mathrm{P}=0,00)$. Con la prueba de Tukey se observa que el tiempo de desarrollo a $25^{\circ}$ y $28^{\circ}$ (Muscidifurax 2,6009 y 2,7565; Spalangia: 2,9353 y 3,1545) fue más corto que de $15^{\circ}$ y $20^{\circ}$ (Muscidifurax 3,2674 y 3,0226; Spalangia 3,4817 y 3,8509; observándose que la duración del ciclo biológico para $S$. endius y Muscidifurax sp. fue significativamente diferente para cada temperatura ensayada.(Tabla 1).El análisis entre ambas especies a una misma temperatura, muestra en todos los casos, una mayor duración del ciclo biológico en $S$. endius, en contraste con Muscidifurax sp. El tiempo de desarrollo a 25 y $28^{\circ} \mathrm{C}$ fue significativamente más corto que a 15 y $20^{\circ} \mathrm{C}$. A la temperatura de $25^{\circ} \mathrm{C}$, la duración del ciclo biológico de $S$. endius fue de 22,60 días y para Muscidifurax sp. 14,80 días (Tabla 2). Considerando ambos sexos a $25^{\circ} \mathrm{C}$, en $S$. endius, las hembras tardaron 24,4 días y los machos 20,8 días y en Muscidifurax sp. las hembras tuvieron un ciclo biológico de 15,6 días y los machos 14 días, datos que coinciden con Moreno (1980)que encontró a $26^{\circ} \mathrm{C}$ un ciclo de 25 días para las hembras de S. endius y 23 para los machos; mientras que para Muscidifurax sp.

Tabla 3. Longevidad según el tipo de microavispa a diferentes temperaturas según la prueba de Tukey

\begin{tabular}{lccccc}
\hline & \multicolumn{5}{c}{ Subconjuto para alfa $=\mathbf{0 , 0 5}$} \\
especie-temperatura & $\mathbf{N}$ & $\mathbf{1}$ & $\mathbf{2}$ & $\mathbf{3}$ & $\mathbf{4}$ \\
\hline Muscidifurax $28^{\circ}$ & 10 & 2,61 & & & \\
Spalangia $28^{\circ}$ & 10 & 2,74 & & & \\
Muscidifurax $25^{\circ}$ & 10 & & 2,97 & & \\
Spalangia $25^{\circ}$ & 10 & & 3,07 & & \\
Spalangia 20 & 10 & & & 3,47 & \\
Muscidifurax $20^{\circ}$ & 10 & & & 3,50 & \\
Muscidifurax $15^{\circ}$ & 10 & & & & 3,74 \\
Spalangia $15^{\circ}$ & 10 & & & & 3,81 \\
Significación & & 0,199 & 0,458 & 0,998 & 0,87 \\
\hline
\end{tabular}

Tabla 4. Prueba de Tukey para longevidad según sexo

\begin{tabular}{lccc}
\hline & $\mathrm{N}$ & 1 & 2 \\
\hline Macho Muscidifurax & 20 & 2,865 & \\
Hembra Muscidifurax & 20 & 2,959 & \\
Macho Spalangia & 20 & & 3,297 \\
Hembra Spalangia & 20 & & 3,415 \\
Significación & & 0,785 & 0,643 \\
\hline
\end{tabular}

fue de 18 días para las hembras y 17 para los machos. Mamani (1984) a $25^{\circ} \mathrm{C}$ reportó 22 días para el ciclo biológico de las hembras de $S$. endius y 21 para los machos.

Longevidad de los parasitoides. La longevidad en ambas especies disminuyó conforme aumentó la temperatura utilizada y está muy asociada con el tipo de alimentación.Esto es confirmado por las ecuaciones de regresión lineal que fueron estadísticamente significativas: $S$. endius $(\mathrm{Y}=77,67-0,99 \mathrm{X} ; \mathrm{F}=731,23$; $\mathrm{gl}=1$ y 2; $\left.\mathrm{R}^{2}=1,00\right)$; Muscidifurax sp. $(\mathrm{Y}=75,99-0,99 \mathrm{X} ; \mathrm{F}=272,00 ; \mathrm{gl}=$ 1 y $\left.2 ; \mathrm{R}^{2}=0,99\right)$, donde $\mathrm{Y}=$ longevidad (días) y $\mathrm{X}=$ temperatura de ensayo. El ANDEVA resultó estadísticamente significativo $(\mathrm{F}=$ 168,83; gl= 7 y 72; P= 0,00). Según la prueba de Tukey,se demuestra que a mayor temperatura los valores de la prueba para longevidad disminuyen (Muscidifurax-28 $8^{\circ}: 2,6131 ; 25^{\circ}: 2,9687 ; 20^{\circ}: 3,5027$; $15^{\circ}: 3,7407$ y Spalangia-28 $8^{\circ}: 2,73 ; 25^{\circ}: 3,0699 ; 20^{\circ}: 3,4718 ; 15^{\circ}$ : $3,8081)$. Se prueba que no existen diferencias significativas entre la longevidad de Muscidifurax y Spalangia, fue diferente con cada temperatura, podemos observar que las longevidades son estadísticamente iguales (Tabla 3). Al evaluar la longevidad en ambos sexos, se observa que para ambas especies de microavispas, (según Tukey Muscidifurax macho: 2,8647, hembra: 2,9590; Spalangia macho 3,2965; hembra: 3,4148) las hembras tienen una mayor longevidad que los machos (Tabla 4) y esta diferencia se acentúa al disminuir la temperatura; así las hembras de $S$. endius viven entre 3 y 5 días más que los machos y en Muscidifurax sp. entre 1 y 8 días. Si son mantenidas a $15^{\circ} \mathrm{C}$, tienen una longevidad promedio de 46,8 y 43,2 días respectivamente, mientras que a $28^{\circ} \mathrm{C}$ sólo viven 16 días y 13,2 días, mientras que a $25^{\circ} \mathrm{C}$, tienen una longevidad promedio de 22 y 24,6 días. Los valores de longevidad obtenidos por Legner y Gerling (1967) señaló 22 días como promedio de la hembra de $S$. endius a 23--27 ${ }^{\circ} \mathrm{C}$, mientras que Mamani(1984) a $25^{\circ} \mathrm{C}$ observó 26 días para las hembras y 19 para los machos coincidiendo con nuestros valores promedio. A diferencia de Moreno (1980) que a $26{ }^{\circ} \mathrm{C}$ encontró una longevidad promedio de 8 días para $S$. endius y 8,5 días para Muscidifurax sp. (Tabla 5).

Capacidad ovipositora. Se comprobó variación a través de los días de exposición de la pupas de $M$. doméstica. $S$. endius tiende a tener una ligera mayor capacidad ovipositora que Muscidifurax sp. y para ambas microavispas, esta capacidad tiende a disminuir con la longevidad de la hembra, esto se evidencia en las ecuaciones de regresión lineal que son estadísticamente significativas: Para $S$.

Tabla 5. Duración promedio de longevidad en días a diferentes temperaturas consideradas en los experimentos con Spalangia endius y Muscidifurax sp. X= Duración promedio de longevidad en días, DS= Desviación Standard

\begin{tabular}{ccccc}
\hline Temperatura & \multicolumn{2}{c}{ Spalangia } & endius & \multicolumn{2}{c}{ Muscidifurax sp. } \\
$\left({ }^{\circ} \mathrm{C}\right)$ & $\mathrm{X}$ & $\mathrm{DS}$ & $\mathrm{X}$ & $\mathrm{DS}$ \\
\hline 15 & 44,20 & $\pm 3,68$ & 41,30 & $\pm 4,03$ \\
20 & 31,40 & $\pm 3,48$ & 32,30 & $\pm 2,63$ \\
25 & 20,70 & $\pm 2,75$ & 18,60 & $\pm 2,41$ \\
28 & 14,60 & $\pm 2,22$ & 12,70 & $\pm 1,34$ \\
\hline
\end{tabular}


Tabla 6. Comparación de la capacidad ovipositora de Spalangia endius y Muscidifurax sp. a $25^{\circ} \mathrm{C}$. X $=$ Promedio de pupas parasitadas, DS= Desviación Standard.

\begin{tabular}{lcccc}
\hline Dias & \multicolumn{2}{c}{ Spalangia } & endius & \multicolumn{2}{c}{ Muscidifurax sp. } \\
& X & DS & X & DS \\
\hline 3 & 18,70 & $\pm 1,16$ & 15,50 & $\pm 1,72$ \\
6 & 12,70 & $\pm 2,95$ & 11,90 & $\pm 3,28$ \\
9 & 12,30 & $\pm 1,70$ & 8,70 & $\pm 2,31$ \\
12 & 7,60 & $\pm 2,46$ & 5,90 & $\pm 1,45$ \\
15 & 9,20 & $\pm 2,78$ & 2,30 & $\pm 1,06$ \\
16 & & & 0,80 & $\pm 0,63$ \\
\hline
\end{tabular}

endius $\left(\mathrm{Y}=19,12-0,94 \mathrm{X} ; \mathrm{F}=114,61 ; \mathrm{gl}=1\right.$ y $\left.14 ; \mathrm{R}^{2}=0,89\right) ; \mathrm{y}$ Muscidifurax sp. $\left(\mathrm{Y}=16,46-0,98 \mathrm{X} ; \mathrm{F}=270,38 ; \mathrm{gl}=1\right.$ y $14 ; \mathrm{R}^{2}=$ $0,95)$, donde $\mathrm{Y}=$ número de pupas parasitadas y $\mathrm{X}=$ longevidad del parasitoide. El ANDEVA fue estadísticamente significativo $(\mathrm{F}=51,48 ; \mathrm{gl}=30$ y $279 ; \mathrm{P}=0,00)$. A partir del día 10 , hubo diferencias significativas en comparación con el tercer día que presentó la máxima capacidad de oviposición. En el grafico de medias marginales, al comparar la capacidad de oviposición de ambas microavispas, se observa que varían a través de los días de exposición, la especie $S$.endius tiende a un ligero incremento en comparación de Muscidifurax (Fig. 1).Además en el día 10 y 15, se observó una mayor capacidad ovipositora de $S$. endius en comparación con Muscidifurax sp. Los demás días, las capacidades ovipositoras de ambas especies fueron similares. Para Muiscidifurax sp., el tercer día se notó la máxima capacidad de oviposición coincidiendo con Mamani (1984) y Polanco (1996). Además a partir del día 7 fue diferente del tercer día de oviposición.

A los 15 días de oviposición en S. endius, alcanzó un promedio de 175 pupas parasitadas, siendo mayor que Muscidifurax sp. el cual parasitó sólo 140 pupas en 16 días, mientras Mamani(1984) obtuvo 196 a $19^{\circ} \mathrm{C}$ y Polanco (1996) 163 a igual temperatura. Generalmente, en ambas especies, la oviposición es de un huevo en el interior de la pupa de la mosca, aunque se encontró hasta cinco huevos, pero únicamente desarrolló uno.

En el caso de $S$. endius, se pudo observar que en los días 9 y 13 , su capacidad ovipositora aumenta numéricamente, sobre Muscidifurax sp. aunque no estadísticamente significativa para luego seguir disminuyendo; esto puede deberse a que $S$. endius puede alcanzar hasta $20 \mathrm{~cm}$ de profundidad y de señalar pupas ya parasitadas. (Tabla 6)

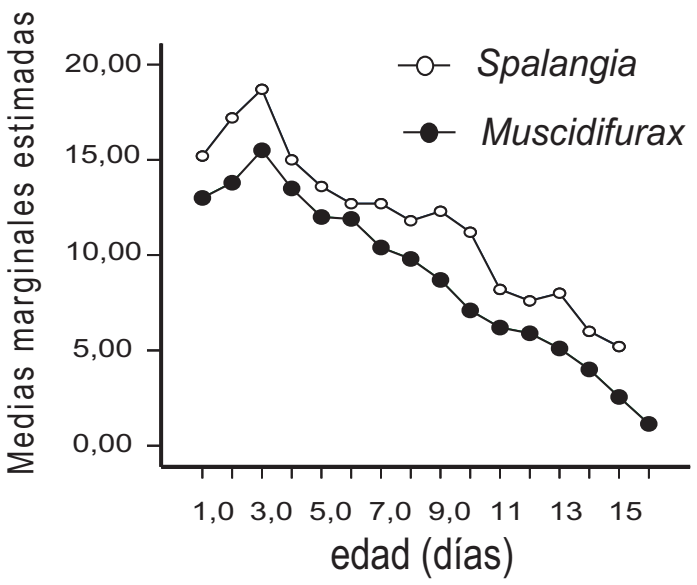

Las medias no estimables no se representan

Figura 1. Medias marginales de capacidad ovopositora. Comparación de Spalangia endius y Muscidifurax sp.

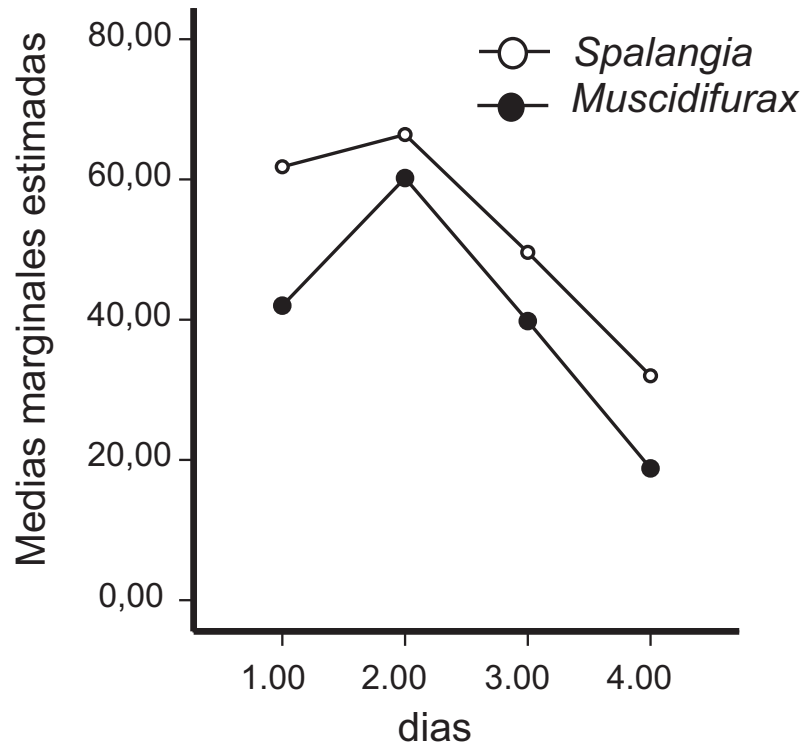

Figura 2. Periodo pupal óptimo. Parasitismo de Spalangia endius y Muscidifurax sp. sobre Musca doméstica.

Determinacion del periodo pupal óptimo de Musca doméstica para el parasitismo. Durante los cuatro estadios pupales de las microavispas se observó que el parasitismo de la pupas varió según el estado de maduración de las mismas; sin embargo las ecuaciones de regresión lineal no fueron estadísticamente significativas: $S$. endius $\left(Y=79,00-0,89 \mathrm{X} ; \mathrm{F}=7,81 ; \mathrm{gl}=1\right.$ y $2 ; \mathrm{R}^{2}=$ 0,8)y Muscidifurax sp. $\left(\mathrm{Y}=62,7-0,69 \mathrm{X} ; \mathrm{F}=1,78 ; \mathrm{gl}=1\right.$ y $2 ; \mathrm{R}^{2}=$ 0,47 ), donde $\mathrm{Y}=$ período de maduración de las pupas (días) y $\mathrm{X}=$ número de pupas parasitadas. Según el ANDEVA, las diferencias son estadísticamente significativas, en relación a la edad de la pupa debido a que el mayor parasitismo ocurre cuando las pupas aún no se esclerotizan y cuando la intensidad de luz es tenue. De acuerdo a las medias marginales se puede observar diferencias significativas para las pupas de 2 días obteniéndose $66,4 \%$ de parasitismo efectivo por $S$. endius y $60,20 \%$ por Muscidifurax sp. (Fig. 2). Morgan et al. (1975) encontró el máximo parasitismo de $S$. endius en pupas de 1 día (25,3\%). El menor parasitismo ocurrió en pupas de 4 días para ambas especies: $32 \%$ en $S$. endius y 18,8\% para Muscidifurax sp., mientras que Moreno (1980) encontró el mínimo en pupas de 3 días (Tabla 7).

Comparación del número óptimo de pupas para exponer con ambas especies. El parasitismo en ambas microavispas estuvo influenciado por el número de pupas expuestas, entre los límites 10 y 50 pupas de mosca/hembra parasitoide. La regresión lineal mostró una tendencia a disminuir el porcentaje de pupas parasitadas con el incremento de pupas expuestas a ambos parasitoides: $S$. endius $\left(Y=94,65-0,76 X ; F=9,29 ; \mathrm{gl}=1\right.$ y $7 ; \mathrm{R}^{2}=$ $0,57)$ y Muscidifurax sp. $(\mathrm{Y}=97,85-0,95 \mathrm{X} ; \mathrm{F}=68,36 ; \mathrm{gl}=11$ y 8 ; $\left.\mathrm{R}^{2}=0,90\right)$ donde $\mathrm{Y}=$ número de pupas parasitadas $\mathrm{y} \mathrm{X}=$ densi-

Tabla 7. Comparación del periodo pupal óptimo de Spalangia endius y Muscidifurax sp. $\mathrm{X}=$ Promedio de pupas Parasitadas, DS= Desviación Standard.

\begin{tabular}{ccccc}
\hline Dias & \multicolumn{2}{c}{ Spalangia endius } & \multicolumn{2}{c}{ Muscidifurax sp. } \\
& $\mathbf{X}$ & DS & X & DS \\
\hline 1 & 61,80 & $\pm 16,69$ & 42,00 & $\pm 8,33$ \\
2 & 66,40 & $\pm 13,36$ & 60,20 & $\pm 13,61$ \\
3 & 49,60 & $\pm 11,65$ & 39,80 & $\pm 10,39$ \\
4 & 32,00 & $\pm 11,59$ & 18,80 & $\pm 7,50$ \\
\hline
\end{tabular}




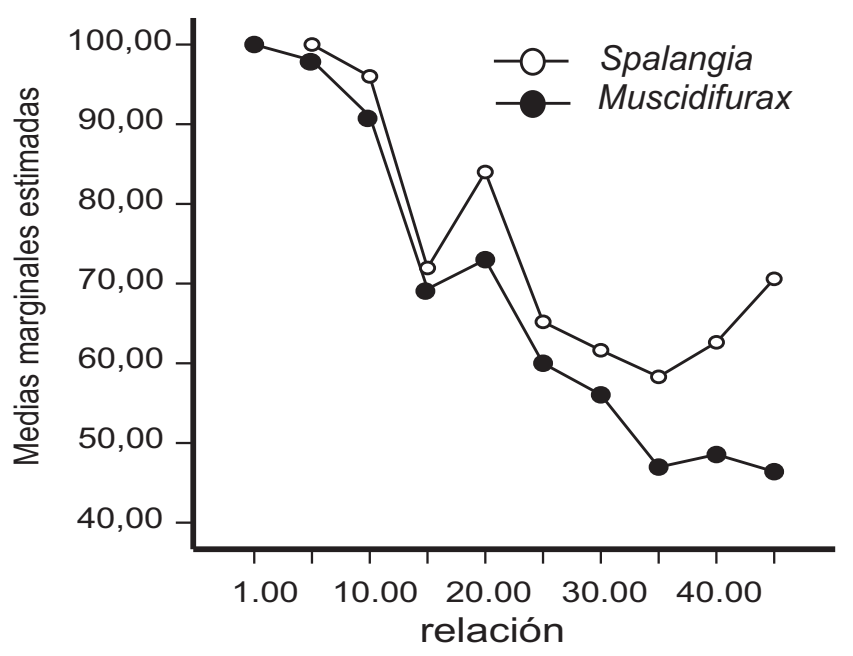

Figura 3. Comparación del número óptimo de pupas de Musca domestica para ambas especies

dad de pupas expuestas. El número óptimo de pupas para exponer a ambos parasitoides fue de 10 (1:10). Observamos en el gráfico de medias marginales (Fig.3) que el porcentaje de pupas expuestas al parasitismo entre los límites de 10 y 50 pupas de mosca/hembra del parasitoide presenta una tendencia a disminuir con el incremento de pupas expuestas a ambos parasitoides. Las relaciones 1:5 y 1:10 fueron diferentes para ambos parasitoides y el análisis de ambas especies mostró que en todas las relaciones no existieron diferencias significativas, excepto en la relación 1:50.Esta relación inversa del parasitismo con respecto al número de pupas expuestas fue hallada también por Ables y Shepard (1974) quienes reportaron para 30 pupas $98 \%$ de parasitismo por S. endius y con 80 pupas $67 \%$ de parasitismo a 25 y $29^{\circ} \mathrm{C}$. (Tabla 8 )

Tabla 8. Análisis comparativo del número óptimo de pupas para exponer a Spalangia endius y Muscidifurax sp. $\mathrm{X}=$ Porcentaje de pupas parasitadas, DS= Desviación Standard.

\begin{tabular}{ccccc}
\hline Relación & \multicolumn{2}{c}{ Spalangia endius } & \multicolumn{2}{c}{ Muscidifurax sp. } \\
& X & DS & X & DS \\
\hline $1: 5$ & 100,00 & $\pm 0,00$ & 98,00 & $\pm 6,32$ \\
$1: 10$ & 96,00 & $\pm 6,99$ & 91,00 & $\pm 11,01$ \\
$1: 15$ & 71,96 & $\pm 14,34$ & 69,33 & $\pm 12,64$ \\
$1: 20$ & 84,00 & $\pm 6,15$ & 73,00 & $\pm 11,35$ \\
$1: 25$ & 65,20 & $\pm 13,60$ & 60,00 & $\pm 9,80$ \\
$1: 30$ & 61,24 & $\pm 10,46$ & 56,04 & $\pm 9,30$ \\
$1: 35$ & 58,30 & $\pm 12,66$ & 46,97 & $\pm 13,88$ \\
$1: 40$ & 62,25 & $\pm 15,70$ & 48,55 & $\pm 12,10$ \\
$1: 50$ & 70,60 & $\pm 13,57$ & 46,40 & $\pm 8,63$ \\
\hline
\end{tabular}

\section{Literatura citada}

Ables,J. y Shepard,M 1974. Responses and competition of the parasitoids Spalangia endius and Muscidifurax raptor (Hymenoptera:Pteromalidae) at different densities of house fly pupas. Canadian Entomologist 106:825-830

Legner, E. y Gerling, D. 1967. Host-feeding and oviposition on Musca domestica by Spalangia cameroni, Nasonia vitripennis and Msucidifurax raptor (Hymenoptera: Pteromalidae) influences theri longevity and fecundity. Ann. Of Entomological Soc. Of America 60: 678-691

Mamani, J. 1984. Efectos de la temperatura en la capacidad reproductiva y en el ciclo de desarrollo de Spalangia endius (Walker)(Hymenoptera:Peteromalidae), parásito pupal de la mosca domestica. Tesis de Maestría. Univ. Nac. Agraria La Molina. Lima-Perú 111p

Moreno,E. 1980. Biología comparada de Muscidifurax sp. y Spalangia endius (Hymenoptera:Pteromalide) ectoparásitos pupales de la mosca doméstica (Musca domestica).Tesis de pre grado Univ. Particular Ricardo Palma. Lima-Perú. 98 pp

Morgan,P.; Patterson R.; Labrecque G.; Weidhaas D. y Benton A. 1975. Suppression of field population of housefly with Spalangia endius. Science 189: 388-389

Ortiz, R y Torres,J. 1983. Ciclo de vida y hábitos de Spalangia cameroni PERKINS (Hymenoptera:Pteromalidae) en condiciones de laboratorio. Tesis de pre grado. Pro-Perkins Ltda. Palmira Colombia. $57 \mathrm{pp}$

Polanco, M. 1996. Crianza de Spalangia endius (Walker) (Hymenoptera: Pteromalidae) parásito pupal de la mosca doméstica en condiciones de laboratorio. Tesis de pre grado. Univ. Nac- San Agustín. Arequipa-Perú 36 pp

Vergara, R. y Jiménez, J. 1995. Manejo integrado de moscas comunes (MIMD) en explotaciones pecuarias y salud pública con énfasis en control biológico. En: Aportes del Control Biológico en la Agricultura Sostenible. RAAA, Lima. Gomero,L y Lizárraga,A. (Eds) 347-359 pp

Zamora, E. 1996. Técnica de producción masiva de Spalangia endius (Walker). II Curso de Actualización en Control Biológico. Centro Nacional de Referencia de Control Biológico. México. $125-129 \mathrm{pp}$ 


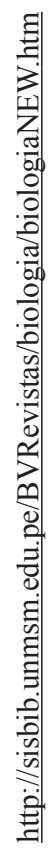

